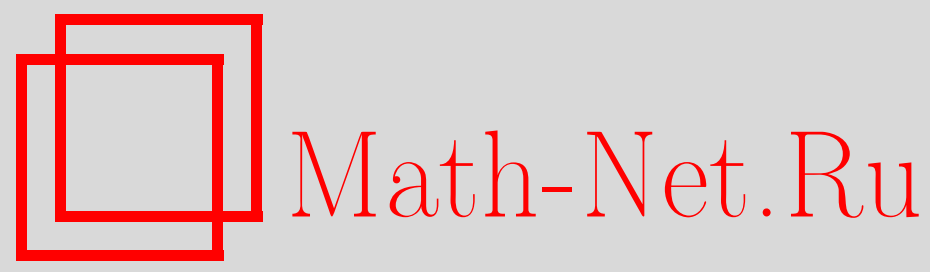

В. А. Коваль, Закон повторного логарифма для матричнонормированных сумм независимых случайных векторов и его применение, Матем. заметки, 2002, том 72, выпуск 3, 363-369

DOI: https://doi.org/10.4213/mzm428

Использование Общероссийского математического портала Math-Net.Ru подразумевает, что вы прочитали и согласны с пользовательским соглашением http://www.mathnet.ru/rus/agreement

Параметры загрузки:

IP: 52.90 .164 .192

26 апреля 2023 г., 05:46:17

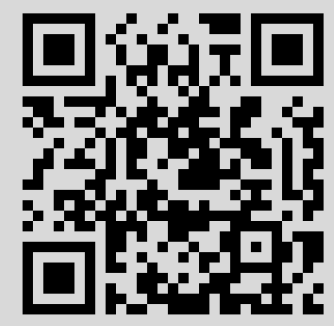




\title{
ЗАКОН ПОВТОРНОГО ЛОГАРИФМА ДЛЯ МАТРИЧНО-НОРМИРОВАННЫХ СУММ НЕЗАВИСИМЫХ СЛУЧАЙНЫХ ВЕКТОРОВ И ЕГО ПРИМЕНЕНИЕ
}

\section{В.А. Коваль}

\begin{abstract}
В работе исследуется закон повторного логарифма в $\mathbb{R}^{d}$ для сумм независимых случайных векторов, которые подвергаются преобразованию матрицами. Рассмотрено применение к многомерной линейной регрессии.

Библиографоя: 10 названий.
\end{abstract}

Пусть $\mathbb{R}^{d}-d$-мерное евклидово пространство вектор-столбцов со скалярным произведением $\langle x, y\rangle=x^{T} y$ и нормой $\|x\|=\sqrt{\langle x, x\rangle}$, где $T$ - знак транспонирования. Норма матрицы $A$ есть $\|A\|=\sqrt{\lambda_{\max }\left(A^{T} A\right)}$, где $\lambda_{\max }(\cdot)$ - максимальное собственное значение матрицы. Если $A$ - симметричная неотрицательно определенная матрица, то $\|A\|=\lambda_{\max }(A)$. Пусть $\left(X_{n}, n \geqslant 1\right)$ - последовательность независимых случайных векторов в $\mathbb{R}^{d}$ с нулевыми средними и $S_{n}=\sum_{i=1}^{n} X_{i}, n \geqslant 1$. В данной работе исследуется закон повторного логарифма (ЗПЛ) для матрично-нормированных сумм $\left(A_{n} S_{n}, n \geqslant 1\right)$, где $\left(A_{n}, n \geqslant 1\right)$ - последовательность матриц размера $q \times d$. Ранее ограниченный ЗПЛ с матричньми нормировками изучался в работе [1].

Рассмотрим сначала нормирование квадратньми корнями $B_{n}^{-1 / 2}$ из обратных матриц $B_{n}^{-1}$ к матрицам $B_{n}=\mathrm{E}\left(S_{n} S_{n}^{T}\right), n \geqslant 1$. Здесь и далее предполагается, что матрица $B_{1}$ невырожденная и, следовательно, при всех $n>1$ определены матрицы $B_{n}^{-1}$. Положим $t_{n}=\sqrt{2 \ln \ln \left\|B_{n}\right\|}$ для достаточно больших $n$.

Теорема 1. 1) Предположим, ито $\lim _{n \rightarrow \infty}\left\|B_{n}\right\|=\infty$ и выполняется условие

$$
\sum_{i=1}^{n} \mathrm{E}\left\|B_{n}^{-1 / 2} X_{i}\right\|^{3}=O\left(\frac{1}{\ln \left\|B_{n}\right\|}\right), \quad n \rightarrow \infty .
$$

Тогда с вероятностью 1

$$
\limsup _{n \rightarrow \infty} \frac{\left\|B_{n}^{-1 / 2} S_{n}\right\|}{t_{n}} \leqslant 1 .
$$

2) Если дополнительно к условиям пункта 1) выполнено условие

$$
\limsup _{n \rightarrow \infty} \frac{\left\|B_{n+1}\right\|}{\left\|B_{n}\right\|}<\infty,
$$

то в (2) имеет место равенство.

Для доказательствапервой части теоремы 1 нам понадобятся следующие три леммы. 
Лемма 1. При всех $m \leqslant n$ имеет место неравенство

$$
\left\|B_{m}^{-1 / 2} B_{n}^{1 / 2}\right\|^{2} \leqslant \frac{\left|B_{n}\right|}{\left|B_{m}\right|}
$$

где $|\cdot|$ - определитель матрицы.

ДокаЗАТЕЛЬСТво. Так как $B_{m} \leqslant B_{n}$ и

$$
B_{m}^{-1 / 2} B_{n} B_{m}^{-1 / 2}=B_{m}^{-1 / 2}\left(B_{n}-B_{m}\right) B_{m}^{-1 / 2}+I,
$$

где $I$ - единичная матрица, то собственные значения $\lambda_{j}$ матрицы $B_{m}^{-1 / 2} B_{n} B_{m}^{-1 / 2}$ удовлетворяют неравенству $\lambda_{j} \geqslant 1, j=1, \ldots, d$. Поэтому

$$
\left\|B_{m}^{-1 / 2} B_{n}^{1 / 2}\right\|^{2}=\lambda_{\max }\left(B_{m}^{-1 / 2} B_{n} B_{m}^{-1 / 2}\right) \leqslant \lambda_{1} \cdots \lambda_{d}=\left|B_{m}^{-1 / 2} B_{n} B_{m}^{-1 / 2}\right|=\frac{\left|B_{n}\right|}{\left|B_{m}\right|} .
$$

Лемма 1 доказана.

Лемма 2. Пусть $Z$ - стандартный гауссовский вектор в $\mathbb{R}^{d}$. Тогда при всех $t>\sqrt{2 d}$ выполнено неравенство

$$
\mathrm{P}(\|Z\|>t) \leqslant \exp \left(-\frac{1}{2}(t-\sqrt{2 d})^{2}\right) .
$$

Лемма 2 есть следствие леммы 3.1 из [2].

ЛЕмма 3. При всех $t>0$ имеет место оценка

$$
\left|\mathrm{P}\left(\left\|B_{n}^{-1 / 2} S_{n}\right\|>t\right)-\mathrm{P}(\|Z\|>t)\right| \leqslant \frac{c}{t^{3}} \sum_{i=1}^{n} \mathrm{E}\left\|B_{n}^{-1 / 2} X_{i}\right\|^{3},
$$

əде с - некоторая абсолютная константа.

Лемма 3 есть следствие теоремы 1 из [3] (см. также п. 6 работы [3]).

ДокАЗАТЕльство теоремы 1. Докажем сначала первую часть теоремы. Последовательность $\left(\left|B_{n}\right|, n \geqslant 1\right)$ монотонно возрастает к бесконечности в силу условия

$$
\lim _{n \rightarrow \infty}\left\|B_{n}\right\|=\infty
$$

и теоремы 3.6.3 [4]. Поэтому для произвольного $\lambda>1$ найдется строго возрастающая последовательность натуральных чисел $\left(n_{j}, j \geqslant 1\right)$, удовлетворяющая неравенствам (см. [5, лемма 3.3])

$$
\lambda\left|B_{n_{j}}\right| \leqslant\left|B_{n_{j+1}}\right| \leqslant \lambda^{3}\left|B_{n_{j}+1}\right| .
$$

В силу леммы Бореля-Кантелли для доказательства (2) достаточно показать, что для всякого $\varepsilon>0$ можно выбрать $\lambda>1$ так, чтобы

$$
\sum_{j=1}^{\infty} \mathrm{P}\left(\max _{n_{j}<n \leqslant n_{j+1}} \frac{\left\|B_{n}^{-1 / 2} S_{n}\right\|}{t_{n}}>1+3 \varepsilon\right)<\infty
$$


(без ограничения общности предполагаем, что $\left\|B_{n_{1}}\right\|>e$ ). Фиксируем $\varepsilon>0$ и положим

$$
\lambda=\left(\frac{1+2 \varepsilon}{1+\varepsilon}\right)^{2 / 3}
$$

Сушествует $j_{0} \geqslant 1$ такое, что $t_{n_{j}}^{2}>2 d \lambda^{3} / \varepsilon^{2}$ при всех $j \geqslant j_{0}$. Заметим, что для любых $m \leqslant n$ из неравенства $B_{m} \leqslant B_{n}$ следует, что $\left\|B_{n}^{-1 / 2} x\right\| \leqslant\left\|B_{m}^{-1 / 2} x\right\|$ при всех $x \in \mathbb{R}$. Воспользуемся неравенством Оттавиани (см. [2, лемма 6.2]), учитывая лемму 1 , правое неравенство (3) и неравенство Чебышева (здесь $j \geqslant j_{0}$ ):

$$
\begin{aligned}
& \mathrm{P}\left(\max _{n_{j}<n \leqslant n_{j+1}} \frac{\left\|B_{n}^{-1 / 2} S_{n}\right\|}{t_{n}}>1+3 \varepsilon\right) \\
& \quad \leqslant \mathrm{P}\left(\max _{n_{j}<n \leqslant n_{j+1}}\left\|B_{n_{j}+1}^{-1 / 2} S_{n}\right\|>(1+3 \varepsilon) t_{n_{j}}\right) \\
& \quad \leqslant \frac{\mathrm{P}\left(\left\|B_{n_{j}+1}^{-1 / 2} S_{n_{j+1}}\right\|>(1+2 \varepsilon) t_{n_{j}}\right)}{1-\max _{n_{j}<n \leqslant n_{j+1}} \mathrm{P}\left(\left\|B_{n_{j}+1}^{-1 / 2}\left(S_{n_{j+1}}-S_{n}\right)\right\|>\varepsilon t_{n_{j}}\right)} \\
& \quad \leqslant \frac{\mathrm{P}\left(\left\|B_{n_{j+1}}^{-1 / 2} S_{n_{j+1}}\right\|>(1+2 \varepsilon) \lambda^{-3 / 2} t_{n_{j}}\right)}{1-\max _{n_{j}<n \leqslant n_{j+1}} \mathrm{P}\left(\left\|B_{n_{j+1}}^{-1 / 2}\left(S_{n_{j+1}}-S_{n}\right)\right\|>\varepsilon \lambda^{-3 / 2} t_{n_{j}}\right)} \\
& \quad \leqslant \frac{\mathrm{P}\left(\left\|B_{n_{j+1}}^{-1 / 2} S_{n_{j+1}}\right\|>(1+\varepsilon) t_{n_{j}}\right)}{1-d \lambda^{3} \varepsilon^{-2} t_{n_{j}}^{-2}} \leqslant 2 \mathrm{P}\left(\left\|B_{n_{j+1}}^{-1 / 2} S_{n_{j+1}}\right\|>(1+\varepsilon) t_{n_{j}}\right) .
\end{aligned}
$$

Таким образом, для доказательства (4) достаточно показать, что

$$
\sum_{j=1}^{\infty} \mathrm{P}\left(\left\|B_{n_{j+1}}^{-1 / 2} S_{n_{j+1}}\right\|>(1+\varepsilon) t_{n_{j}}\right)<\infty
$$

В силу леммы 3 это соотношение будет выполнено, если

$$
\sum_{j=1}^{\infty} \frac{1}{t_{n_{j}}^{3}} \sum_{i=1}^{n_{j+1}} \mathrm{E}\left\|B_{n_{j+1}}^{-1 / 2} X_{i}\right\|^{3}<\infty \quad \text { и } \quad \sum_{j=1}^{\infty} \mathrm{P}\left(\|Z\|>(1+\varepsilon) t_{n_{j}}\right)<\infty .
$$

В свою очередь, в силу условия (1) и леммы 2 для этого достаточно соответственно показать, что

$$
\sum_{j=1}^{\infty} \frac{1}{\left(\ln \ln \left\|B_{n_{j}}\right\|\right)^{3 / 2} \ln \left\|B_{n_{j+1}}\right\|}<\infty
$$

и

$$
\sum_{j=1}^{\infty}\left(\ln \left\|B_{n_{j}}\right\|\right)^{-(1+\varepsilon)^{2}\left(1-\delta_{j}\right)^{2}}<\infty
$$

где $\delta_{j} \rightarrow 0$ при $j \rightarrow \infty$. Используя левое неравенство (3), получаем оценку $\left\|B_{n_{j}}\right\| \geqslant$ $\lambda^{(j-1) / d}\left|B_{n_{1}}\right|^{1 / d}, j \geqslant 1$. Отсюда следуют соотношения (5) и (6). 
Перейдем к доказательству второй части теоремы. Так как

$$
\left\|B_{n}^{-1 / 2} x\right\| \geqslant \frac{\|x\|}{\left\|B_{n}^{1 / 2}\right\|}
$$

для любого $x \in \mathbb{R}^{d}$, для доказательства соотношения

$$
\limsup _{n \rightarrow \infty} \frac{\left\|B_{n}^{-1 / 2} S_{n}\right\|}{t_{n}} \geqslant 1 \quad \text { с вероятностью } 1
$$

достаточно показать, что с вероятностью 1

$$
\limsup _{n \rightarrow \infty} \frac{\left\|S_{n}\right\|}{\left\|B_{n}\right\|^{1 / 2} t_{n}} \geqslant 1 .
$$

Из условий (1) и

$$
\limsup _{n \rightarrow \infty} \frac{\left\|B_{n+1}\right\|}{\left\|B_{n}\right\|}<\infty
$$

следует выполнение условия (1.20) работы [6]. Поэтому в силу [6, теорема 1.2] имеет место (7).

Теорема 1 доказана.

Заметим, что в одномерном случае $(d=1)$ теорема 1 совпадает с теоремой 4 в работе [7, гл. 17].

ЗАмЕчАнИЕ 1. Из доказательства теоремы 1 следует, что в гауссовском случае условие (1) не требуется.

Пусть далее $\left(A_{n}, n \geqslant 1\right)$ - произвольная последовательность матриц размера $q \times d$ $\left(A_{n} \neq 0, n \geqslant 1\right)$.

Теорема 2. Пусть $\lim _{n \rightarrow \infty}\left\|B_{n}\right\|=\infty$ и выполнено условие (1). Тогда с вероятностью 1

$$
\limsup _{n \rightarrow \infty} \frac{\left\|A_{n} S_{n}\right\|}{\left\|A_{n} B_{n} A_{n}^{T}\right\|^{1 / 2} t_{n}} \leqslant 1 .
$$

ДокАЗАТЕЛЬСТво следует из теоремы 1 в силу неравенства

$$
\left\|A_{n} S_{n}\right\| \leqslant\left\|A_{n} B_{n}^{1 / 2}\right\| \cdot\left\|B_{n}^{-1 / 2} S_{n}\right\|
$$

и тождества

$$
\left\|A_{n} B_{n}^{1 / 2}\right\|=\left\|A_{n} B_{n} A_{n}^{T}\right\|^{1 / 2} .
$$

Заметим, что матрица $A_{n} B_{n} A_{n}^{T}$ является ковариационной матрицей случайного вектора $A_{n} S_{n}$.

Для выяснения условий, при которых в соотношении (8) имеет место точное равенство, ограничимся для простоты гауссовским случаем, что позволяет, кроме того, накладьвать наименее жесткие ограничения на последовательность $\left(A_{n}, n \geqslant 1\right)$. Введем обозначение

$$
a_{n}=\left\|A_{n} B_{n} A_{n}^{T}\right\|^{1 / 2}, \quad n \geqslant 1 .
$$

Обозначим через $\left(e_{n}, n \geqslant 1\right)$ последовательность единичных векторов в $\mathbb{R}^{q}$, для которых $A_{n} B_{n} A_{n}^{T} e_{n}=a_{n}^{2} e_{n}, n \geqslant 1$. 
Теорема 3. Пусть $\left(X_{n}, n \geqslant 1\right)$ - последовательность независимых центрированных гауссовских случайных векторов в $\mathbb{R}^{d}$ и выполнено следующее условие: найдется постоянная $c>0$ и последовательность положительных чисел $\left(f_{n}, n \geqslant 1\right)$ такая, что

$$
\begin{gathered}
\lim _{n \rightarrow \infty} f_{n}=\infty, \\
\limsup _{n \rightarrow \infty} \frac{f_{n+1}}{f_{n}}<\infty, \\
\ln \ln f_{n} \sim \ln \ln \left\|B_{n}\right\|, \quad n \rightarrow \infty,
\end{gathered}
$$

и при всех $n>m \geqslant 1$ выполняется неравенство

$$
\frac{\left\langle B_{m} A_{m}^{T} e_{m}, A_{n}^{T} e_{n}\right\rangle}{a_{m} a_{n}} \leqslant c \frac{f_{m}}{f_{n}} .
$$

Тогда с вероятностью 1

$$
\limsup _{n \rightarrow \infty} \frac{\left\|A_{n} S_{n}\right\|}{a_{n} t_{n}}=1
$$

ДоКАЗАТЕЛЬСтво. Из (9) и (11) следует, что $\lim _{n \rightarrow \infty}\left\|B_{n}\right\|=\infty$. Поэтому в силу теоремы 2 и замечания 1 с вероятностью 1

$$
\limsup _{n \rightarrow \infty} \frac{\left\|A_{n} S_{n}\right\|}{a_{n} t_{n}} \leqslant 1
$$

Покажем, что с вероятностью 1

$$
\limsup _{n \rightarrow \infty} \frac{\left\|A_{n} S_{n}\right\|}{a_{n} t_{n}} \geqslant 1
$$

Рассмотрим центрированную гауссовскую последовательность

$$
\left(\zeta_{n}=\left\langle A_{n}^{T} e_{n}, S_{n}\right\rangle, \quad n \geqslant 1\right)
$$

Заметим, что ее дисперсия $\mathrm{E} \zeta_{n}^{2}=a_{n}^{2}$, а коэффициент корреляции

$$
r_{m, n}=\frac{\left\langle B_{m} A_{m}^{T} e_{m}, A_{n}^{T} e_{n}\right\rangle}{a_{m} a_{n}}, \quad n>m \geqslant 1 .
$$

В силу условий (9), (10) и (12) на основании пункта 2) теоремы из работы [8] заключаем, что с вероятностью 1

$$
\limsup _{n \rightarrow \infty} \frac{\left|\left\langle A_{n}^{T} e_{n}, S_{n}\right\rangle\right|}{a_{n} \sqrt{2 \ln \ln f_{n}}} \geqslant 1 .
$$

Так как $\left\|A_{n} S_{n}\right\| \geqslant\left|\left\langle A_{n}^{T} e_{n}, S_{n}\right\rangle\right|, n \geqslant 1$, то из (11) и (14) следует (13).

Теорема 3 доказана. 
В качестве примера применения теоремы 3 исследуем скорость сходимости оценок наименьших квадратов неизвестного параметра многомерной линейной регрессии с гауссовскими возмущениями. Аналогично, используя теорему 2, можно рассмотреть более общую ситуацию. Пусть результаты наблюдений $\left(Y_{n}, n \geqslant 1\right)$ имеют следующую структуру:

$$
Y_{n}=\Phi_{n}^{T} \theta+Z_{n}, \quad n \geqslant 1
$$

где $\left(Z_{n}, n \geqslant 1\right)$ - последовательность независимых центрированных гауссовских случайных векторов в $\mathbb{R}^{d}$ с общей ковариационной матрицей $\sigma^{2} I$ ( $\sigma^{2}$-положительное число); $\left(\Phi_{n}, n \geqslant 1\right)$ - последовательность матриц размера $d \times q ; \theta$ - неизвестный параметр. Предположим, что матрицы $\psi_{n}=\sum_{j=1}^{n} \Phi_{j} \Phi_{j}^{T}, n \geqslant 1$, невырождены. Тогда оценки наименьших квадратов $\widehat{\theta}_{n}$ параметра $\theta$ имеют следующий вид:

$$
\widehat{\theta}_{n}=\psi_{n}^{-1} \sum_{i=1}^{n} \Phi_{i} Y_{i}
$$

Если минимальные собственные значения матриц $\psi_{n}$ удовлетворяют условию

$$
\lim _{n \rightarrow \infty} \lambda_{\min }\left(\psi_{n}\right)=\infty
$$

то с вероятностью 1

$$
\left\|\widehat{\theta}_{n}-\theta\right\| \rightarrow 0, \quad n \rightarrow \infty
$$

(см. [9, теорема 3]). Исследуем скорость сходимости в (15).

СлЕДСТВИЕ 1. Пусть выполнены следующие условия:

$$
\begin{gathered}
\lim _{n \rightarrow \infty} \lambda_{\min }\left(\psi_{n}\right)=\infty, \quad \limsup _{n \rightarrow \infty} \frac{\lambda_{\min }\left(\psi_{n+1}\right)}{\lambda_{\min }\left(\psi_{n}\right)}<\infty, \\
\ln \ln \lambda_{\min }\left(\psi_{n}\right) \sim \ln \ln \lambda_{\max }\left(\psi_{n}\right), \quad n \rightarrow \infty
\end{gathered}
$$

Тогда с вероятностью 1

$$
\limsup _{n \rightarrow \infty}\left(\frac{\lambda_{\min }\left(\psi_{n}\right)}{2 \sigma^{2} \ln \ln \lambda_{\min }\left(\psi_{n}\right)}\right)^{1 / 2}\left\|\widehat{\theta}_{n}-\theta\right\|=1
$$

ДокАЗАТЕльство. Так как $\widehat{\theta}_{n}-\theta=\psi_{n}^{-1} \sum_{i=1}^{n} \Phi_{i} Z_{i}$, для доказательства $(16)$ нужно проверить условия теоремы 3 , в которой $A_{n}=\psi_{n}^{-1}, B_{n}=\sigma^{2} \psi_{n}, a_{n}=\sigma / \sqrt{\lambda_{\min }\left(\psi_{n}\right)}$ и векторы $\left(e_{n}, n \geqslant 1\right)$ удовлетворяют соотношению $\psi_{n}^{-1} e_{n}=\left(\lambda_{\min }\left(\psi_{n}\right)\right)^{-1} e_{n}$. Нетрудно убедится, что условие (12) выполнено с $c=1$ и $f_{n}=\sqrt{\lambda_{\min }\left(\psi_{n}\right)}, n \geqslant 1$. Вьполнение условия (11) очевидно.

Следствие доказано.

Отметим, что скорость сходимости в (15) покоординатно исследовалась в работах [9], $[10]$. 


\section{СПИСОК ЦИТИРОВАННОЙ ЛИТЕРАТУРЫ}

[1] Buldygin V., Solntsev S. Asymptotic Behaviour of Linearly Transformed Sums of Random Variables. Dordrecht: Kluwer, 1997.

[2] Ledoux M., Talagrand M. Probability in Banach Spaces. Berlin: Springer, 1991.

[3] Ротарь В. И. Неравномерная оценка скорости сходимости в многомерной центральной пределной теореме // Теория вероятн. и ее примен. 1970. Т. 15. № 4. С. 647-665.

[4] Ланкастер П. Теория матриц. М.: Наука, 1978.

[5] Wittmann R. A general law of iterated logarithm // Z. Wahrscheinlichkeit. Verw. Gebiete. 1985. V. 68. № 4. P. 521-543.

[6] Chen X. On the law of the iterated logarithm for independent Banach space-valued random variables // Ann. Probab. 1993. V. 21. № 4. P. 1991-2011.

[7] Боровков А. А. Теория вероятностей. М.: Наука, 1986.

[8] Коваль В.О. Про закон повторного логарифма для гауссових послідовностей та його застосування // Теор. ймовірност. матем. статист. 1996. № 54. С. 66-71.

[9] Lai T. L. Some almost sure convergence properties of weighted sums of martingale difference sequences // Almost Everywhere Convergence II (Evanston, IL, 1989). Boston: Acad. Press, 1991. P. 179-190.

[10] Lai T. L., Wei C.Z. A law of the iterated logarithm for double arrays of independent random variables with applications to regression and time series models // Ann. Probab. 1982. V. 10. № 2. P. 320-335.

Национальный технический университет Украины (КПИ), г. Киев

Поступило

E-mail: luda@com.zt.ua

03.10.2001

Исправленный вариант

13.02.2002 